\title{
Sequence-based detection of mutations in cadherin 1 to determine the prevalence of germline mutations in patients with invasive lobular carcinoma of the breast
}

\author{
Allyson L Valente ${ }^{1}$, Seth Rummel ${ }^{1}$, Craig D Shriver ${ }^{2}$ and Rachel E Ellsworth ${ }^{3^{*}}$
}

\begin{abstract}
Background: Loss of cadherin 1 (CDH1) expression, which is normally involved in cell adhesion and maintenance of tissue architecture, is a hallmark of invasive lobular carcinoma (ILCA). Because hereditary cancers may require different risk reduction, counseling and treatment options than sporadic cancer, it is critical to determine the prevalence of germline $\mathrm{CDH} 1$ mutations in patients with ILCA.

Methods: All patients with ILCA $(n=100)$ previously enrolled in the Clinical Breast Care Project were identified. Genomic DNA was isolated from peripheral blood samples and DNA variants were detected for each exon of $\mathrm{CDH1}$ using high-resolution melting technology followed by direct sequencing.

Results: Within the 100 samples screened, four nonsynonymous variants were detected: A592T in one Hispanic patient, A617T in two patients, both African American, P825L in a Causasian patient whose grandmother had stomach cancer, and G879S in a Caucasian patient. Further evaluation of A617T in an additional 165 African American patients found that 11 patients, none with ILCA, carried this variant including one patient who was homozygous for the variant.

Conclusions: $\mathrm{CDH} 1$ mutations are infrequent in patients with ILCA, and the variants that were detected have been classified as non-pathogenic. These data suggest that ILCA does not have a significant hereditary component and do not support $\mathrm{CDH} 1$ gene mutation testing in patients with ILCA.
\end{abstract}

Keywords: Breast cancer, ILCA, CDH1

\section{Background}

Breast cancer represents a group of diseases, with different histological, molecular and clinical characteristics, including tumor growth patterns, with at least 17 different histological types currently described [1]. Invasive lobular carcinoma (ILCA), the second most common histological type, accounts for $8-14 \%$ of all breast cancers [2,3]. ILCA is histologically characterized by a loss of cellular adhesion, resulting in growth of single cells or cells arranged in a single file or "Indian lines" [3,4]. ILCA is often bilateral, multifocal and multicentric, and

\footnotetext{
* Correspondence: r.ellsworth@wriwindber.org

${ }^{3}$ Clinical Breast Care Project, Henry M. Jackson Foundation for the Advancement of Military Medicine, 620 Seventh Street, Windber, PA, USA Full list of author information is available at the end of the article
}

is more difficult to detect by palpation or mammography [5,6]. Compared to invasive ductal carcinoma (IDCA), ILCA is more often diagnosed at a later age, more likely to be hormone receptor positive, larger, diploid, and HER2, p53 and EGFR negative. ILCA has a distinctive pattern of metastasis, with a propensity to metastasize to the peritoneum, gastrointestinal tract and ovaries. Despite its favorable characteristics, disease-free and overall survival did not differ between ILCA and IDCA [7].

One of the hallmarks of ILCA is the loss or altered expression of cadherin 1 (CDH1, GenBank: AB025105.1). $\mathrm{CDH} 1$ is a member of the classical/type I cadherin family that is involved in maintaining cellular adhesion. CDH1 interacts with members of the catenin family; this complex then links with actin molecules to provide cellular adhesion 
[8]. Altered expression of CHD1 explains the discohesive phenotype characteristic of not only ILCA but also hereditary diffuse gastric cancer (HDGC). In fact, ILCA is a frequent finding in HDGC families, with a 39\% lifetime risk of developing ILCA in HDGC families with CDH1 germline mutations [9].

The frequency of $\mathrm{CDH} 1$ germline mutations in families with HDGC is $25-30 \%$ [10]. Determination of the frequency of CDH1 mutations in patients with ILCA is crucial; clinically, the diffuse nature of ILCA makes early detection more challenging [5] and increased risk for bilateral disease impacts the choice of surgical approaches. In addition, presence of hereditary $\mathrm{CDH} 1$ mutations will impact risk assessment, family counseling and screening, for both breast and stomach malignancies. To this end, $\mathrm{CDH} 1$ was screened for germline mutations in a population of patients with ILCA.

\section{Methods}

All patients included in this study were enrolled in the Clinical Breast Care Project (CBCP) between 2001 and 2010. Blood samples were collected with approval from the Walter Reed National Military Medical Center Human Use Committee and Institutional Review Board. All subjects voluntarily agreed to participate and gave written informed consent. Clinical information, including family cancer history, was obtained for all CBCP participants using questionnaires designed by and administered under the auspices of the CBCP.
The CBCP database was queried to identify all female patients diagnosed with ILCA. Family history of cancers was self-described by the patients and included data for both primary and secondary relatives. Diagnosis of every specimen was performed by a dedicated breast pathologist from hematoxylin and eosin (H\&E) stained slides; staging was performed using guidelines defined by the AJCC Cancer Staging Manual seventh edition [11]. In addition, to determine the frequency of the A617T variant in African American women, all female African Americans with invasive breast cancers such as IDCA and tumors with mixed IDCA and ILCA features were identified.

Genomic DNA was isolated from blood clots using the GentraClotspin and Puregene DNA purification kits (Qiagen, Valencia, CA). Polymerase chain reaction (PCR) primers were designed to flank $\mathrm{CDH} 1$ exons $1-15$ and the coding region of exon 16 (Table 1 ). PCR reactions were performed on $25 \mathrm{ng}$ genomic DNA using Lightscanner Master Mix (BioFire Defense, Salt Lake City, UT) and analyzed on the LightScanner System following manufacturer's protocols. For those samples with a variant detected by the LightScanner, PCR products were purified using ExoSAP-IT For PCR Product Clean-up (Affymetrix, Santa Clara, CA), and sequenced using BigDye terminator v 3.1 cycle sequencing kits (Life Technologies, Inc, Carlsbad, CA). The resulting products were sequenced on a 3730xl DNA Analyzer (Life Technologies, Inc, Carlsbad, CA) and sequence files were analyzed using Sequencher 4.10.1 (Gene Codes Corporation, Ann Arbor, MI).

Table 1 PCR primers and annealing temperature for each of the 16 exons

\begin{tabular}{|c|c|c|c|}
\hline Coding region & Forward sequence & Reverse sequence & Annealing temperature \\
\hline Exon 1 & ggtgcctccggggctca & gaatgcgtccctcgcaagt & $70.5^{\circ} \mathrm{C}$ \\
\hline Exon 2 & gagtcacccggttccatctacctt & ccagggacaccggcagc & $70.5^{\circ} \mathrm{C}$ \\
\hline Exon 3 & tcttgtctttaatctgtccaatttcctaatctc & gcgcactaaaacaacagcga & $63.5^{\circ} \mathrm{C}$ \\
\hline Exon 4 & tatccgtcttgaattgtcttatcttgttcctcatc & ccctcccagagaaacagagaactt & $63.5^{\circ} \mathrm{C}$ \\
\hline Exon 5 & cagtgttgggatccttctttactaattc & cccggtgtcaacaagcttctaa & $63.5^{\circ} \mathrm{C}$ \\
\hline Exon 6 & tttcttcctcatcagagctcaagt & tccaaagaacctaagagtctttctgagta & $69.0^{\circ} \mathrm{C}$ \\
\hline Exon 7 & tcccaaagtgcagcttgtctaa & taatacacatttgtcctccacaccc & $60.5^{\circ} \mathrm{C}$ \\
\hline Exon 8 & gtcctgacttggttgtgtcg & agacctttctttggaaaccctctaa & $69.0^{\circ} \mathrm{C}$ \\
\hline Exon 9 & agtcttggtactttgtaaatgacacatct & agctgtgaggatgccagtt & $69.0^{\circ} \mathrm{C}$ \\
\hline Exon 10 & aaatgtttcgttttgtttttaacttcattgt & ccagttgctgcaagtcagttga & $67.5^{\circ} \mathrm{C}$ \\
\hline Exon 11 & tttcagctacatgttgtttgctggtcctatt & gctaggaggtcgaggcagcaaag & $60.5^{\circ} \mathrm{C}$ \\
\hline Exon 12 & ttgccaagctgccacattt & gcatggcagttggagcaaag & $63.5^{\circ} \mathrm{C}$ \\
\hline Exon 13 & cctcccctggtctcatcatttc & aagtcaaaggctgagtcacttgc & $67.5^{\circ} \mathrm{C}$ \\
\hline Exon 14 & cttctttatctttggctctcaacactt & agagatcaccactgagctacc & $63.5^{\circ} \mathrm{C}$ \\
\hline Exon 15 & cctactcttcattgtacttcaacct & tgagcttagagatgagccatgc & $63.5^{\circ} \mathrm{C}$ \\
\hline Exon 16.1 & acaggtgtgeccttcctttcactaa & ccaccagcaacgtgatttctgcattt & $60.5^{\circ} \mathrm{C}$ \\
\hline
\end{tabular}

Cycling conditions were: $95.0^{\circ} \mathrm{C}$ for two minutes, followed by 50 cycles of $94.0^{\circ} \mathrm{C}$ for 30 seconds and the specified annealing temperature for 30 seconds, followed by a single cycle of $94.0^{\circ} \mathrm{C}$ for 30 seconds and then $20.0^{\circ} \mathrm{C}$ for 30 seconds. 
The A617T variant was investigated using TaqMan SNP genotyping assay C_32307311_10 (Life Technologies, Inc, Carlsbad, CA) using $10 \mathrm{ng}$ of DNA; reactions were amplified in duplicate and genotypes (TT, TC or CC) were determined using the ABI PRISM ${ }^{\circledast} 7000 \mathrm{Se}$ quence Detection System software (Applied Biosystems, Foster City, CA).

\section{Results}

\section{Clinicopathological characteristics}

Of the 100 patients with ILCA, six had a self-reported history of gastric or stomach cancer in primary or secondary members of their family. None of the patients had a personal history of any type of cancer other than ILCA. The average age at diagnosis was 61.7 years (range 44-70 years). The majority of patients were Caucasian (83\%), with early-stage (86\%) and ER + HER2(93\%) lobular carcinomas. Thirty-two percent of the patients had a family history of breast cancer, defined as at least two primary or secondary relatives having been diagnosed with breast cancer at any age, while $20 \%$ of patients had bilateral breast cancer, similar to that seen in other ILCA populations [7]. Two of the patients, both diagnosed with stage IIIC luminal A ILCA have died of disease.

\section{Mutation results}

No nonsense or splice site mutations were detected in any of the 100 genomic DNAs evaluated. A number of SNPs were detected in multiple individuals, including seven synonymous variants, two intronic variants and one variant in the 5' UTR (Table 2). Four missense mutations were identified including private variant P825L, detected in a 62 year old white woman whose grandmother had stomach cancer, A592T in a 51 year-old white woman, A617T in two African Americans, both post-menopausal, and G879S in an 81 year-old white woman.

With the identification of A617T in two African American women, the variant was assessed in a panel of genomic DNA samples from 165 additional African American women with IDCA or mixed features of IDCA and ILCA. Ten women were carriers of the minor (T) allele and one additional patient, an African American woman diagnosed with with IDCA at age 71, was homozygous for the minor allele. The frequency of the $\mathrm{T}$ allele in this population was similar to that reported in dbSNP (http://www.ncbi.nlm.nih.gov/projects/SNP/snp_ref.cgi?rs= 33935154) where it has been detected only in Sub-Saharan African and African American populations.

\section{Discussion}

Identification of patients with hereditary predisposition to breast cancer is critical for optimum clinical management.
Table 2 Summary of variants in patients with ILCA

\begin{tabular}{|c|c|c|c|}
\hline SNP ID & SNP effect & $\begin{array}{l}\text { Number of patients with } \\
\text { variant }(n=100)\end{array}$ & Ethnicity $^{\mathrm{a}}$ \\
\hline c. $71 \mathrm{C}>\mathrm{G}$ & 5' UTR & 3 & W \\
\hline $345 G>A$ & Synonymous & 1 & W \\
\hline $\begin{array}{l}531+10 G> \\
C\end{array}$ & Intronic & 9 & W \\
\hline $933 C>G$ & Synonymous & 1 & $\mathrm{AA}$ \\
\hline $1680 G>C$ & Synonymous & 1 & W \\
\hline A592T & Missense & 1 & $\mathrm{HS}$ \\
\hline A617T & Missense & 1 & $\mathrm{AA}$ \\
\hline $1896 C>T$ & Synonymous & 2 & $\mathrm{AA}$ \\
\hline $2076 \mathrm{~T}>\mathrm{C}$ & Synonymous & 46 & $\begin{array}{l}\text { W, AA, HS, } \\
\text { NA }\end{array}$ \\
\hline 1937-13 T>C & Intronic & 6 & W \\
\hline $95971 \mathrm{C}>\mathrm{T}$ & Synonymous & 6 & W \\
\hline P825L & Missense & 1 & W \\
\hline G879S & Missense & 1 & W \\
\hline $101193 C>A$ & Synonymous & 2 & $\mathrm{AA}$ \\
\hline
\end{tabular}

${ }^{\mathrm{a}}$ Ethnicity groups are: $\mathrm{AA}=$ African American, $\mathrm{HS}=$ Hispanic, $\mathrm{NA}=$ Native American, $\mathrm{W}=$ White

Identification of carriers of causative mutations can assist in risk assessment and risk reduction, as well as in family counseling. In addition, hereditary cancers may have different phenotypes, requiring different treatment regimens. For example, patients with BRCA1 mutations are at risk for both bilateral breast disease as well as ovarian carcinoma; thus, women with BRCA1-associated cancers may be offered bilateral mastectomy and/or oophorectomy and may derive more benefit from PARP inhibitors compared to patients with sporadic breast cancer [12]. Patients with both hereditary and sporadic forms of ILCA may benefit from targeted therapeutics such as the use of FGFR1 inhibitors that block survival pathways and reverse tamoxifen resistance [13], however, those with hereditary ILCA may, as those with BRCA1 and BRCA2 mutations, be subjected to increased screening for bilateral disease, offered risk reduction strategies, as well encouraged to undergo prophylactic gastrectomy [14].

The frequency of potentially causative mutations in this study was low, with no instances of splice site or nonsense mutations detected. Only 5\% (5/100) of patients had missense mutations. Under current guidelines in which genetic testing for BRCA1 and BRCA2 mutation is recommended for women with estimated probabilities of having a germline mutation $\geq 10 \%$ [15], genetic testing for CDH1 mutations in patients with ILCA would not be warranted. In addition, the clinical importance of the variants detected is unclear. To determine the pathogenicity of these variants, the pubmed database (http:// www.ncbi.nlm.nih.gov/pubmed/) was searched for any citations that included these variants. In addition, the 
Catalog of Somatic Mutations in Cancer (COSMIC) database (http://cancer.sanger.ac.uk/cancergenome/projects/cosmic/) was searched. To our knowledge, variants P825L and G879S have not been documented as causative mutations in the literature, although a single instance of the P825L variant in a lung tumor was reported in the COSMIC database. Functional assessment of A592T suggests this variant is likely tolerable and non-pathogenic [16]. The A617T variant has been detected in a patient with endometrial cancer [17] and in two female African American patients with diffuse gastric cancer [18]. Transfection of $\mathrm{CHO}$ cells with $\mathrm{CDH} 1$ carrying the A617T variant resulted in an epithelial phenotype with extensive cell-to-cell contacts with membranous E-cadherin and $\beta$ catenin staining similar to that in wild-type cells [19]. Data from our study supports A617T as being a non-pathogenic variant; 10/165 African American women with IDCA and one with mixed IDCA and ILCA were carriers of the minor allele. In addition, one 71 year-old woman with IDCA was homozygous for the minor allele. The $6.7 \%$ minor allele frequency for A617T detected in our patient samples is identical to that reported in dbSNP for African American populations; thus this variant likely represents a nonpathogenic SNP specific to individuals with African ancestry. In addition, we performed a SIFT (Sorting Intolerant From Tolerant) [20] analysis for the four non-synonymous variants. A592T, A617T and G879S were classified as tolerable variants, while P825L was predicted to be intolerable.

Our ability to detect mutations may be limited by the technical approach employed. Large deletions may not be detected by high-resolution melting analysis or direct sequencing of amplified products, however, the reported frequency of large-scale deletions in HDGC families is low (3.8\%) [21], thus it is not likely that many of the ILCA patients have an undetected deletion. In addition, genomic DNA samples from family members were not collected, thus, it was not possible in this study to determine if the variants tracked with disease status. Finally, identification of patients with a family history of gastric cancer was based on self-reporting of cancer within first- and second-degree family members by the patient and ages at diagnosis were not provided. Of the $100 \mathrm{pa}-$ tients with ILCA, six had a single self-reported family member with stomach or gastric cancer, thus, the majority of these patients may not have a family history of HDGC.

\section{Conclusions}

In our data of patients with ILCA unselected for age at diagnosis or family history of breast cancer, the mutation frequency was low - four missense mutations, only one of which (P825L) has been predicted to be pathogenic in five individuals. These patients were recruited from a breast, rather than gastroenterology clinic, and thus the mutational frequency and spectrum differs based on presence or absence of a significant family history of HDGC. A previous study of 318 women with ILCA diagnosed $<45$ years or with a family history of breast cancer found no deletions or truncating mutations and of the six detected non-synonymous mutations, only four were found to be pathogenic, for a mutation frequency of $1.3 \%$ [22]. Together, these data suggest that the frequency of germline mutations in patients with ILCA is low, and screening for CDH1 mutations in this population is not warranted.

\section{Competing interests}

The authors declare that they have no competing interests.

\section{Authors' contributions}

ALV performed the high-resolution melting and sequence analysis and reviewed the manuscript, SR performed the genotyping of the A617T variant and reviewed the manuscript, CDS oversaw collection of all patient samples and reviewed the manuscript, REE designed the project, performed data analysis and wrote the manuscript. All authors read and approved the final manuscript.

\section{Acknowledgements}

This research was supported by a grant from the United States Department of Defense (Military Molecular Medicine Initiative MDA W81XWH-05-2-0075, Protocol 01-20006). The opinion and assertions contained herein are the private views of the authors and are not to be construed as official or as representing the views of the Department of the Army or the Department of Defense.

\section{Author details}

${ }^{1}$ Clinical Breast Care Project, Windber Research Institute, 620 Seventh Street, Windber, PA, USA. ${ }^{2}$ Clinical Breast Care Project, Walter Reed National Military Medical Center, 8901 Wisconsin Ave, Bethesda, MD, USA. ${ }^{3}$ Clinical Breast Care Project, Henry M. Jackson Foundation for the Advancement of Military Medicine, 620 Seventh Street, Windber, PA, USA.

Received: 14 April 2014 Accepted: 11 July 2014

Published: 19 July 2014

\section{References}

1. Weigelt B, Geyer FC, Reis-Filho JS: Histological types of breast cancer: how special are they? Mol Oncol 2010, 4:192-208.

2. Borst MJ, Ingold JA: Metastatic patterns of invasive lobular versus invasive ductal carcinoma of the breast. Surgery 1993, 114:637-641.

3. Martinez V, Azzopardi JG: Invasive lobular carcinoma of the breast: incidence and variants. Histopathology 1979, 3:467-488.

4. Fisher ER, Gregorio RM, Fisher B, Redmond C, Vellios F, Sommers SC: The pathology of invasive breast cancer: a syllabus derived from findings of the National Surgical Adjuvant Breast Project (protocol no. 4). Cancer 1975, 36:1-85.

5. Krecke KN, Gisvold JJ: Invasive lobular carcinoma of the breast: mammographic findings and extent of disease at diagnosis in 184 patients. AJR Am J Roentgenol 1993, 161:957-960.

6. Lesser ML, Rosen PP, Kinne DW: Multicentricity and bilaterality in invasive breast carcinoma. Surgery 1982, 91:234-240.

7. Arpino G, Bardou VJ, Clark GM, Elledge RM: Infiltrating lobular carcinoma of the breast: tumor characteristics and clinical outcome. Breast Cancer Res 2004, 6:R149-R156.

8. Paredes J, Figueiredo J, Albergaria A, Oliverira P, Carvalho J, Ribeiro AS, Caldeira J, Costa AM, Simoes-Correia J, Oliveira MJ, Pinheiro H, Pinho SS, Mateus R, Reis CA, Leite M, Ferdandes MS, Schmitt F, Carneiro F, Figueiredo C, Oiveira C, Seruca R: Epithelial E- and P-cadherins: role and clinical significance in cancer. Biochem Biophys Res Commun 1826, 2012:297-311.

9. Pharoah PD, Guilford P, Caldas C: Incidence of gastric cancer and breast cancer in $\mathrm{CDH} 1$ (E-cadherin) mutation carriers from hereditary diffuse gastric cancer families. Gastroenterology 2001, 121:1348-1353. 
10. Fitzgerald RC, Hardwick R, Huntsman D, Cameiro F, Guilford P, Blair V, Chung DC, Norton J, Ragunath K, Van Krieken JH, Dwerryhouse S, Caldas C, International Gastric Cancer Linkage Consortium: Hereditary diffuse gastric cancer: updated consensus guidelines for clinical management and directions for future research. J Med Genet 2010, 47:436-444.

11. American Joint Committee on Cancer: AJCC Cancer Staging Manual. 7th edition. New York: Springer; 2010.

12. Silver DP: PARP Inhibition in Triple Negative Breast Cancer. 2011. 8 November, 2011.

13. Sikora MJ, Cooper KL, Bahreini A, Luthra S, Wang G, Chandran UR, Davidson $N E$, Dabbs DJ, Welm AL, Oesterreich S: Invasive lobular carcinoma cell lines are characterized by unique estrogen-mediated gene expression patterns and altered tamoxifen response. Cancer Res 2014, 74:1463-1474.

14. Kluijt I, Sijmons RH, Hoogerbrugge N, Plukker JT, de Jong D, Van Krieken JH, van Hillegersberg RL, Ligtenberg M, Bleiker E, Cats A, Dutch Working Group on Hereditary Gastric Cance: Familial gastric cancer: guidelines for diagnosis, treatment and periodic surveillance. Fam Cancer 2012, 11:363-369.

15. Statement of the American Society of Clinical Oncology: genetic testing for cancer susceptibility. J Clin Oncol 1996, 14:1730-1736.

16. Garziera M, Canzoneiri V, Cannizzaro R, Geremia S, Caggiari L, De Zorzi M, Maiero S, Orzes E, Perin T, Zanussi S, de Paoli P, De Re V: Identification and characterization of $\mathrm{CDH} 1$ germline variants in sporadic gastric cancer patients and in individuals at risk of gastric cancer. PlOS ONE 2013 8:e77035.

17. Risinger Jl, Berchuck A, Kohler MF, Boyd J: Mutations of the E-cadherin gene in human gynecologic cancers. Nat Genet 2014, 7:98-102.

18. Suriano G, Oliveira C, Ferreira P, Machado JC, Bordin MC, De Wever O, Bruyneel EA, Moguilevsky N, Grehan N, Porter TR, Richards FM, Hruban RH, Roviello F, Huntsman D, Mareel M, Carneiro F, Caldas C, Seruca R: Identification of $\mathrm{CDH} 1$ germline missense mutations associated with functional inactivation of the E-cadherin protein in young gastric cancer probands. Hum Mol Genet 2014, 12:575-582

19. Suriano G, Oliveira MJ, Huntsman D, Mateus AR, Ferreira P, Casares F, Oliveira C, Cameiro F, Machado JC: E-cadherin germline missense mutations and cell phenotype: evidence for the independence of cell invasion on the motile capabilities of the cells. Hum Mol Genet 2003, 12:3007-3016

20. Ng PC, Henikoff S: SIFT: predicting amino acid changes that affect protein function. Nucleic Acids Res 2003, 31:3812-3814.

21. Oliveira C, Senz J, Kaurah P, Pihheiro H, Sanges R, Haegert A, Corso G, Schouten J, Fitzgerald R, Vogelsang H, Keller G, Dwerryhouse S, Grimmer D, Chin SF, Yang HK, Jackson CE, Seruca R, Roviello F, Stupka E, Caldas C, Huntsman D: Germline CDH1 deletions in hereditary diffuse gastric cancer families. Hum Mol Genet 2009, 18:1545-1555.

22. Schrader KA, Masciari S, Boyd N, Salamanca C, Senz J, Saunders DN, Yorida E, Maines-Bandiera S, Kaurah P, Tung N, Robson ME, Ryan PD, Olopade OI, Domchek SM, Ford J, Issacs C, Brown P, Balmana J, Razzak AR, Miron P, Coffey K, Terry MB, John EM, Andrulis IL, Knight JA, O'Malley FP, Daly M, Bender P, KConFab, Moore R et al: Germline mutations in $\mathrm{CDH} 1$ are infrequent in women with early-onset or familial lobular breast cancers. J Med Genet 2011, 48:64-68.

doi:10.1186/1897-4287-12-17

Cite this article as: Valente et al:: Sequence-based detection of mutations in cadherin 1 to determine the prevalence of germline mutations in patients with invasive lobular carcinoma of the breast. Hereditary Cancer in Clinical Practice 2014 12:17.

\section{Submit your next manuscript to BioMed Central and take full advantage of:}

- Convenient online submission

- Thorough peer review

- No space constraints or color figure charges

- Immediate publication on acceptance

- Inclusion in PubMed, CAS, Scopus and Google Scholar

- Research which is freely available for redistribution

Submit your manuscript at www.biomedcentral.com/submit
C Biomed Central 